of intersection of the parabolic cylinders (3) and (4) and a sphere the radius of which is equal to the momentum $(P)$ of the incident beam. (These curves also lie on the circular cylinders $p_{x}^{2}+\left(p_{y}+n a h / 2 \pi\right)^{2}=$ $P^{2}+2 m E_{r}$ where $n=1$ and 2.)

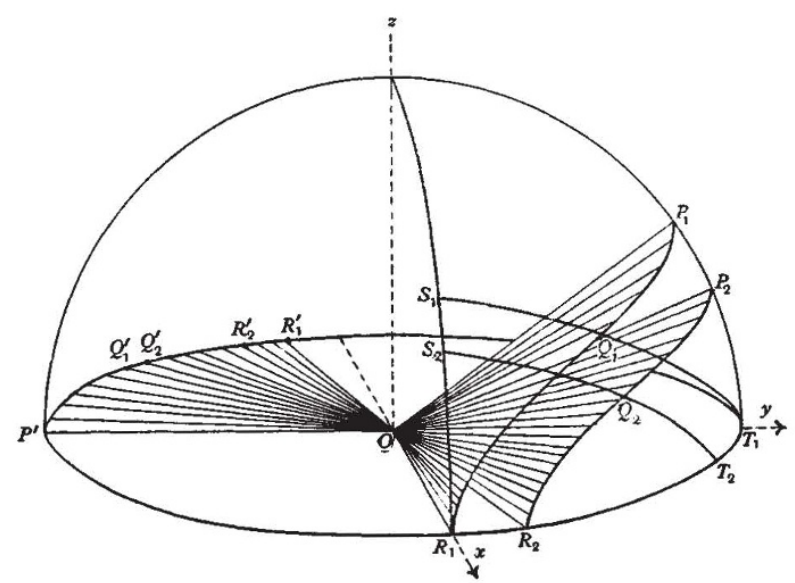

FIG. 2. The critical directions for selective adsorption.

The directions given by (3) are illustrated for a particular case in Fig. 2. The directions of the lines joining the curves $P_{1} R_{1}, P_{2} R_{2}, \ldots$ to the origin are the critical directions. For each such incident direction there is a diffracted beam $O P^{\prime}, O R^{\prime}$... in the plane of the crystal. There are similar critical directions $S_{1} T_{1}, S_{2} T_{2} \ldots$ obtained by changing $p_{y}$ to $p_{x}$ in the equations (3) and (4). The direction $O Q_{1}$, where $Q_{1}$ is the point of intersection of $P_{1} R_{1}$ and $S_{1} T_{1}$, is doubly critical, for atoms may then be selectively adsorbed in two ways. As the radius of the sphere changes, the point $Q_{1}$ will trace out a parabola in the plane $p_{x}=p_{y}$. A tangent to this parabola will give the direction where the most pronounced effect may be expected, when a nonmonochromatic beam is used, provided the mean energy has the right value.

The fact that the theory here given is successful in explaining the observations suggests that helium atoms moving with the energy of the beams which Stern and Frisch used (corresponding to a temperature of $300^{\circ}$ absolute) migrate freely along the surface. It seems reasonable, too, to infer that the inverse process takes place in Nature. Atoms moving along the surface with the right energy and in the right direction may be diffracted so as to leave the surface with positive energy and thus be evaporated. This is a new mechanism of evaporation which has not pre. viously been suspected.

University Chemical Laboratory

J. E. LENNARD-JONES. A. F. Devonshire. Cambridge.

${ }^{2}$ Frisch and Stern, Z. Phys., 84, 430 and 443 (1933).

\section{Raman Spectrum of Gaseous and Liquid Sulphur Dioxide and its Solutions in Water}

THE Raman spectrum of gaseous sulphur dioxide at a pressure of 12 atmospheres was determined with large dispersion (Hilger $E_{1}$ spectrograph). Only the strong frequency $1150 \cdot 5 \pm 0.5$ was found, whereas Bhagavantam ${ }^{1}$ gives 1154. The two weak, diffuse lines of the molecule could not be obtained, probably owing to the fairly strong background inevitable in very long exposures.

The frequencies of the liquid were redetermined, and the influence of the temperature on them was studied at $30^{\circ}$ and $80^{\circ}$. Only a small displacement of the strong, sharp line towards higher values, with increasing temperature, was found $(1144 \cdot 3 \pm 0 \cdot 2$ at $30^{\circ} ; 1144.9 \pm 0.3$ at $\left.80^{\circ}\right)$. No influence on the broad and diffuse lines $524 \cdot 5 \pm 1$ and $1336 \cdot 0 \pm 1$ was perceptible.

For the frequencies of the $\mathrm{SO}_{2}$ molecule in water solutions at $60^{\circ}$ the values $531 \pm 1$ (0, br., diff.), $1150 \cdot 0 \pm 0.5 \quad(4$ sharp $)$ and $1331 \pm 1\left(\frac{1}{2}, d\right)$ were obtained. No lines attributable to the ions $\mathrm{SO}_{3}$ " or $\mathrm{HSO}_{3}{ }^{\prime}$ were found. (As the solution, although it was freed from oxygen and kept in an evacuated, sealed tube, became yellow owing to disinte. gration, very long exposures were impossible.) It is interesting to note that the larger part of the $\mathrm{SO}_{2}$ molecules in the solution are present as such, and that the frequency of the stronger Raman line agrees nearly completely with that of the gaseous $\mathrm{SO}_{2}$ itself. The state of the molecules in the solution is apparently comparable with that in the free gas.

\section{H. Gerding.}

Laboratory of Inorganic and Physical Chemistry,

University, Amsterdam. May 11.

${ }^{1}$ NATURB, 128, 995 (1930).

\section{Artificial Radioactivity giving Continuous $\gamma$-Radiation}

IN many cases of nuclear transformations, unstable nuclei are obtained which break down emitting positive electrons ( $\beta+$-decay). Because this nucleus is surrounded by orbital negative electrons, the positive electron produced can recombine with one of the negatives giving rise to a $\gamma$-ray quantum ${ }^{1}$. The force responsible for recombination is proportional to $e$ (electronic charge). The probability of such a recombination thus involves the factor $e^{2}$. Therefore the ratio of the probability of observing a $\gamma$-ray quantum to that of finding a positive electron must be of the order of magnitude $e^{2} / h c \sim 10^{-2}\left(e^{2} / h c\right.$ being the only dimensionless number which can be formed from $e$, the Planck constant $h$ and the velocity of light $c$ ).

We conclude that any $\beta+$-emission must be accom. panied by a weak $\gamma$-radiation. In analogy to the theory of optical dispersion, we can consider the decayed nucleus plus the (positive and negative) electrons as an intermediate state. If a transition from the initial state (non-decayed nucleus plus orbital electrons) to the final state (decayed nucleus plus a $\gamma$-ray quantum) is energetically possible, such a transition can occur even if the energy is too small to reach the intermediate state (decayed nucleus plus positive electron plus orbital electrons).

Artificial $\beta+$-activity occurs, if the energy difference between the decayed and the undecayed nucleus is greater than $m c^{2}$. Emission of a continuous $\gamma$-ray spectrum (not accompanied by $\beta+$-emission) should be observed if this energy difference is less than $m c^{2}$, but greater than $-m c^{2}$. The mean life of such a $\gamma$-active nucleus is about a thousand times longer than that of a $\beta+$-active nucleus. It seems worth while to look for this new form of radioactivity. 